\title{
Dynamical phasing of Type II Cepheids
}

\author{
J.A. McSaveney \\ Mount Stromlo Observatory, Research School of Astronomy and \\ Astrophysics, Australian National University, Canberra, Australia \\ K.R. Pollard, P.L. Cottrell \\ Mount John University Observatory, Department of Physics and \\ Astronomy, University of Canterbury, Christchurch, New Zealand
}

\begin{abstract}
By phasing using the minimum of the acceleration curve of a selection of Type II Cepheids of a range of periods, a clearer understanding of the pulsational dynamics of the stars is obtained. BVRI photometry and a selection of metallic line velocities and $\mathrm{H} \alpha$ equivalent widths are presented using this phasing.
\end{abstract}

\section{Introduction}

Traditional phasing of Cepheid light and radial velocity curves has been based on the time of light maximum or minimum. This leads to ambiguities as the positioning of such extrema depend on the filter region used. The phase of minimum light occurs earliest in $B$, with the minima occurring later in the redder filters for SW Tau, $\kappa$ Pav (Fig. 1), W Vir and RU Cen (Fig. 2). Also, in the case of stars such as W Vir (Fig. 2a), there is an initial peak in the light curve which is strongest in the blue and decreases in amplitude at redder wavelengths, such that the corresponding peak in $I$ may not be the maximum light value. These variations can result in problems comparing stars, especially with respect to examining at which phase particular spectral features appear.

An alternative approach is called for which allows comparison of the same physical processes at the same phases for the stars examined. While examining the acceleration curve of the FeI lines of SW Tau, it was noted that the acceleration minimum was sharper and more clearly defined than the light minimum previously used. It was decided to use this to phase the available data and extend the technique to other stars.

\section{Theory and technique}

The advantage of using the acceleration minimum is that it gives a common phasing point representative of the minimum photospheric radius of the lineforming region used. This means that it is based on a much more narrowlydefined region than that of the photometry. By placing the time of minimum radius of a relatively narrow region of the star at phase 0.0 , a clear zero-point 

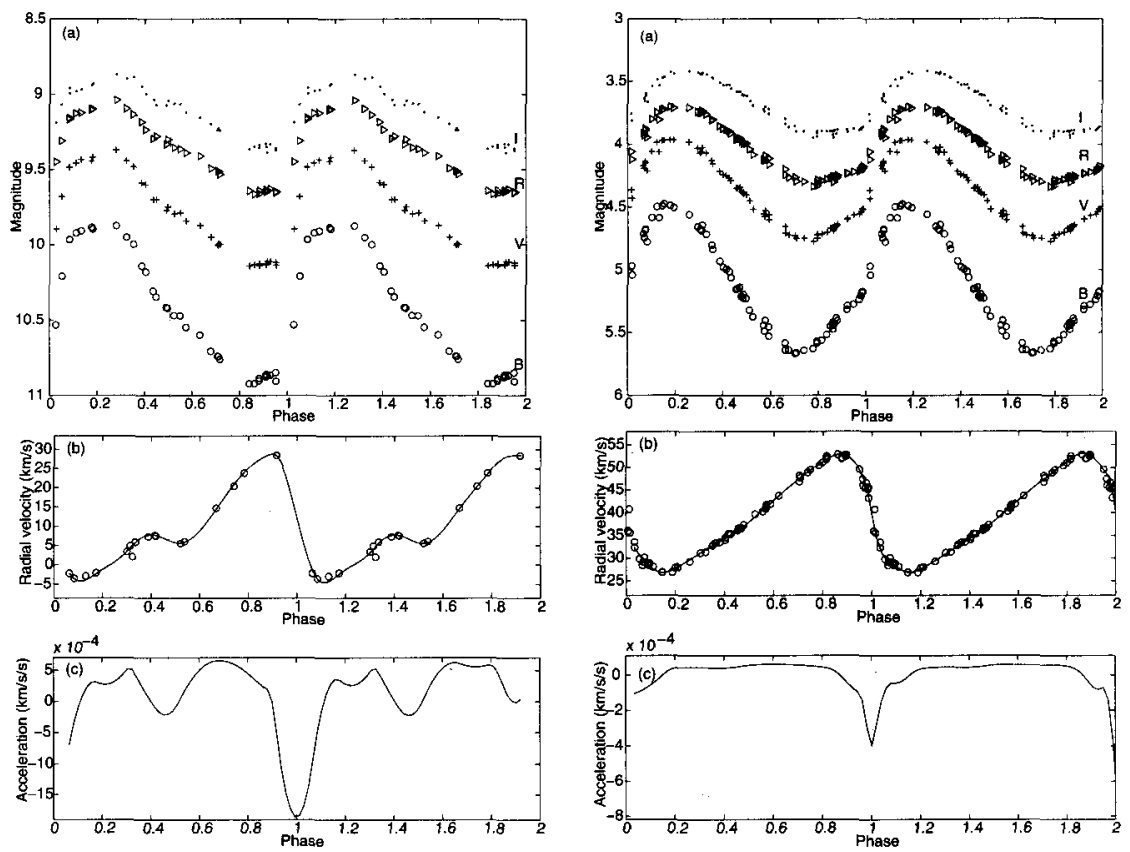

Figure 1. Left: SW Tau. Right: $\kappa$ Pav (a) BVRI photometry; (b) spline-fitted mean FeI radial velocities; and (c) acceleration curves. SW Tau is phased on a 1.583560 -d period. $\kappa$ Pav is phased on a 9.0714d period.

is used which is easily compared between stars. This zero-point represents the boundary in time between the bulk of the material in the line-forming region falling inward and the bulk of the material being forced outwards by the layers underneath. This can occur as a smooth change in mean motion, as observed in SW Tau and $\kappa$ Pav (Fig. 1b), or abruptly in association with the passage of a shock wave through the line-forming region, as observed in W Vir and RU Cen (Fig. 2b).

A spline curve was fitted to the mean Fe I radial velocity curve of SW Tau (Fig. 1b) and, from the derivative of the spline, an acceleration curve was derived (Fig. 1c). This technique was repeated on a selection of BL Her, W Vir and RV Tauri stars (McSaveney 2003) of which SW Tau, $\kappa$ Pav, W Vir and RU Cen are presented here. In the case of RU Cen, which has weak or non-existent Fe I lines, the stronger $\mathrm{Ba} I \mathrm{II}$ lines were used instead.

\section{Results and discussion}

All the stars show clear acceleration minima from which to phase the data (Figs 1c and 2c). As RU Cen has been phased on the period between successive deep minima, it shows two acceleration minima per cycle and has been phased 

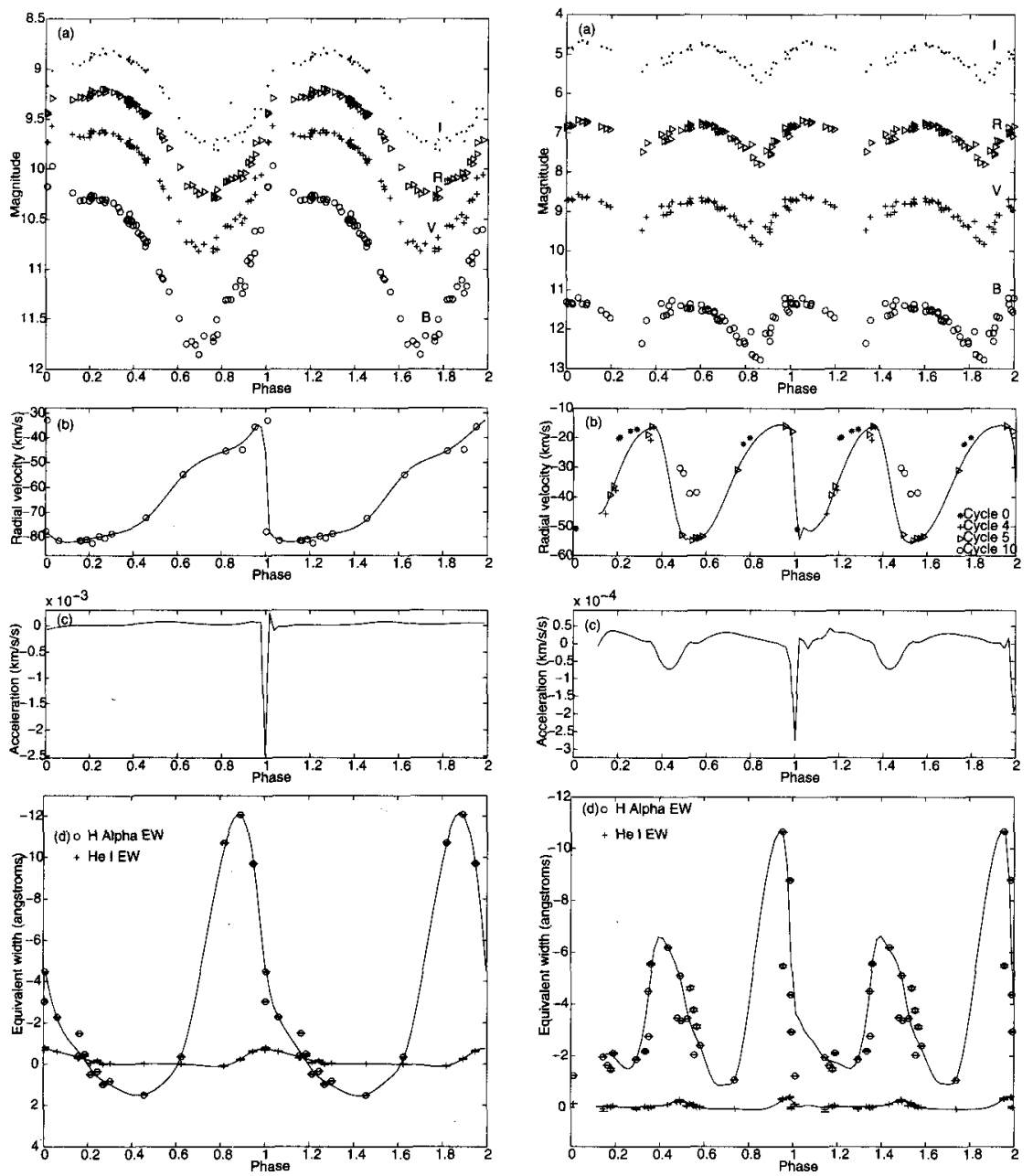

Figure 2. Left: W Vir. Right: RU Cen (a) BVRI photometry; (b) spline-fitted mean Fe I radial velocities for W Vir (left) and Ba II radial velocities for RU Cen (right); (c) acceleration curves; and (d) $\mathrm{H} \alpha$ and He I equivalent widths. W Vir is phased on a $17.2768-d$ period. RU Cen is phased on a $64.57-\mathrm{d}$ period. 
with respect to the deeper of the two. In this case, the deeper acceleration minimum occurs just prior to the deeper light curve minimum and peak $\mathrm{H} \alpha$ emission. The acceleration minimum also coincides with peak HeI emission. Both these emission features, and the very sharp acceleration minimum, are associated with the passage of a strong shock wave through the line-forming regions. These shock features also occur in a slightly weaker form in association with the shallower light minimum around phase 0.5 .

Similar shock features following similar phase patterns are also observed in W Vir (Fig. 2), although only one shock per pulsation cycle is visible. Such strong shock features are not observed in SW Tau and $\kappa$ Pav (Fig. 1) although both stars show line splitting associated with weaker shocks in their $\mathrm{H} \alpha$ line absorption profiles (McSaveney 2003). Based on the acceleration phasing, these shocks occur at the same phases as the stronger shocks observed in W Vir and RU Cen.

As with the different minima occurring at different phases in the light curves, the acceleration minima positions vary depending on the line species used. This is a result of the different lines being formed over slightly different regions of the stellar atmosphere, and therefore reaching minimum photospheric radius at slightly different times. It is, however, less of a problem in comparison to the shift between photometric filters since the line-forming regions are more narrowly defined so that there is less ambiguity with respect to the physics of the system. This should also make it easier to compare with modelled data.

\section{Conclusion}

The acceleration minimum has provided an excellent reference point for phasing the spectroscopic and photometric data from a selection of Type II Cepheids. Phasing with respect to this minimum has meant that the stars are phased based on the minimum photospheric radius of the line-forming region and so share a common, more physically defined, zero-point than that of light curve minima. This has allowed a comprehensive comparison of the shock effects observed in the stars.

A systematic pattern has emerged, where the light minimum is followed by peak $\mathrm{H} \alpha$ emission in W Vir and RU Cen. The acceleration minimum follows and is observed to occur at the same phase as shock-related line splitting and peak HeI emission. While the strength and presence of these feature varies with the strength of the associated shock, the order of appearance seems not to vary. Future work includes applying the technique to a greater range of stars to see if the same results hold and to extend the comparisons to models of the stars.

Acknowledgments. This work is based on part of JAM's PhD thesis at the University of Canterbury. Spectra were obtained by JAM but thanks go to Alan Gilmore and Pam Kilmartin who obtained the BVRI photometry.

\section{References}

McSaveney, J.A. 2003, Dynamics of Type II Cepheids, PhD thesis, University of Canterbury 